\title{
RESEARCH ON HISTORIC BIM OF BUILT HERITAGE IN TAIWAN -A CASE STUDY OF HUANGXI ACADEMY
}

\author{
Y. C. Lu ${ }^{1, a}$, T. Y. Shih ${ }^{1, b}$, Y. N. Yen ${ }^{2, c}$ \\ ${ }^{1}$ National Chiao Tung University, Civil Engineering, Hsinchu, Taiwan \\ ${ }^{a}$ kevin801006.cv03g@nctu.edu.tw \\ b tyshih@mail.nctu.edu.tw \\ ${ }^{2}$ Dept. of Architecture, China University of Technology, Taipei, Taiwan \\ ${ }^{\mathrm{c}}$ alexyen@cute.edu.tw
}

Commission II, WG II/8

KEY WORDS: BIM, historic BIM, object-oriented class diagram, ontology, historical database

\begin{abstract}
:
Digital archiving technology for conserving cultural heritage is an important subject nowadays. The Taiwanese Ministry of Culture continues to try to converge the concept and technology of conservation towards international conventions. However, the products from these different technologies are not yet integrated due to the lack of research and development in this field. There is currently no effective schema in HBIM for Taiwanese cultural heritage. The aim of this research is to establish an HBIM schema for Chinese built heritage in Taiwan. The proposed method starts from the perspective of the components of built heritage buildings, up to the investigation of the important properties of the components through important international charters and Taiwanese laws of cultural heritage conservation. Afterwards, object-oriented class diagram and ontology from the scale of components were defined to clarify the concept and increase the interoperability. A historical database was then established for the historical information of components and to bring it into the concept of BIM in order to build a 3D model of heritage objects which can be used for visualization. An integration platform was developed for the users to browse and manipulate the database and 3D model simultaneously. In addition, this research also evaluated the feasibility of this method using the study case at the Huangxi academy located in Taiwan. The conclusion showed that class diagram could help the establishment of database and even its application for different Chinese built heritage objects. The establishment of ontology helped to convey knowledge and increase interoperability. In comparison to traditional documentation methods, the querying result of the platform was more accurate and less prone to human error.
\end{abstract}

\section{INTRODUCTION}

\subsection{Research background}

The development of digital archiving technology is rapidly changing the mode of conserving heritage buildings. The International Council on Monuments and Sites (ICOMOS) strongly suggested people to apply these technologies to the conservation of heritage sites widely. Some conditions in the application of world heritage are integrated to digital archiving technology (Yen, 2014); In addition, some researchers applied the technology of Building Information Modelling (BIM) to manage the life cycle of heritage buildings.

The Taiwanese government tries to converge the concept and technology of conservation towards international conventions. However, the concepts is quite difficult to reach the artisans or workers who directly execute the repairing works of heritage buildings (Fu, 2005).

There are many databases for historic buildings in Taiwan designed by the Taiwanese government, such as the National Cultural Heritage Database Management System, the Cultural Heritage Department of Taichung City, and the Department of Cultural Affairs of the Taipei City Government. All of these examples are built for storing and sharing historical information of buildings. None of the existing database has detailed information for every component within built heritage buildings which is the basic information for BIM.

BIM refers to a technology which can produce digital information and manage the life cycle of the target building. In another word, BIM is a technology, a method and a concept which can assist the life cycle of a building in terms of its layout, design, construction, management and repairs based on a manual process in a virtual space (Hsieh et al., 2014). Due to
BIM's advances in management and visualization, BIM has seen applications in the conservation and repair of built heritage buildings, thus creating the Historic Building Information Modeling (HBIM).

Taiwan has many kinds of Chinese traditional buildings, especially the Minnan style. With the evolution times, many artisans who were proficient at the crafts of these styles no longer exists and less people are willing to carry on the skills. Traditionally, artisans passed on their crafts orally or demonstrated the crafts on site. It is difficult for someone to document or learn the crafts, resulting in the lost knowledge of many precious crafts. In the future, we may encounter the situation where no one knows how to repair or maintain the historic buildings when it is necessary.

Due to the misunderstanding of relational laws and the wrong conception for maintaining and repairing since a long time, many artisans in Taiwan thought that a repairing work of built heritage building means that one must make it perfect as so people cannot recognize that it has been repaired. Basically, the main concern of conservation and repairing should be the authenticity and the historical nature of the object. To investigate what attributes of building components are related to its authenticity and historical nature is one of the aims in this research.

The purpose of this research is to develop an HBIM schema for Chinese built heritage building in Taiwan. The proposed method starts from the perspective of the components of the built heritage buildings, up to the investigation of the important properties of the components through important international charters and Taiwanese laws of cultural heritage 
conservation. Afterwards, object-oriented class diagram and ontology from the scale of components were defined to clarify the concept and increase the interoperability. A historical database was then established for the historical information of components and to bring it into the concept of BIM in order to build a 3D model of heritage objects which can be used for visualization. An integration platform was developed for the users to browse and manipulate the database and 3D model simultaneously.

\section{LITERATURE REVIEW}

\subsection{Historic BIM (HBIM)}

HBIM is an application of the BIM technology for assisting the conservation or repairs of built heritage buildings (Murphy et al., 2007). Different from modern buildings, the original blueprint of historic building might not exist anymore. In addition, some buildings have many complex components for decoration or architectural purposes. The creation of building component models in BIM software without its blueprint and complex surfaces poses a particular problem (Oreni et al., 2013).

Apart from building the 3D model of a building component, it is also important to consider the historical information of components. In fact, the artistic values in built heritage buildings often carries deep meaning within local culture and can pass down the culture effectively. For example, in the case of a decoration component of a building, it is possible to extract the fabrication method, name of the artisan, and the meaning beyond the components' geometric properties. Unfortunately, those attribute fields does not exist in current BIM software (Autodesk Revit ${ }^{1}$, 2017). It is important to conserve the historical information with the geometric model together. In this regard, there are many researches focusing on the method or process of assisting the conservation of historical information in architectural components by using HBIM (Oreni et al., 2014).

Many research in the HBIM domain for Chinese traditional built heritage buildings discusses about the final precision of 3D model of buildings or the process of acquiring the model. However, the historical information of components is less discussed although it is also important. This research aims to develop a schema for the archiving of a Chinese historic building in which the historical information and the $3 \mathrm{D}$ model can be browsed simultaneously.

\subsection{Object-Oriented Class Diagram}

An object is a basic logical unit within Object-Oriented Programming (OOP). Every object is an individual for a specific class and a class can relate to other classes through inheritance, aggregation and so on (Tsai, 2009). The concept of OOP can be analogous to BIM and architectural components, in which a component is an object and a class is used to collect different types of components. It is possible to explore the relationship between different classes through the method used in OOP.

The Industry Alliance for Interoperability (IAI) applied OOP to develop the Industry Foundation Classes (IFC). IFC is made to improve the interoperability of the information in the domain of architecture and related industries. It is used to compose a computer readable model which includes data elements that represent parts of buildings and their relevant information (EI-Mekawy, 2012). In the standard document completed by IAI and ISO, the ISO 16739 is a model used to present the concept of class as can be seen in Figure 1. This standard demonstrates the possibility of establishing the building components and the relationship of the classes through the concept of OOP.

This research attempted to build a class diagram for components of historic building by using the concept of OOP in order to make a detailed description of the value of attribute and the structure within components. A clearer overview on the concept of Chinese historic building and its components is expected as the result of this research.

\subsection{Ontology}

Ontology is used for describing and expressing knowledge in certain professional domains. It describes knowledge of the domain conceptually through clear and formal definition or description. Ontology is a model which demonstrates the concept that the conveyance of knowledge is affected by the design of model and it is also used to describe the relationship between concepts (Pattuelli, 2011). There is no standard (ISO like) for ontology respected by all users at this moment (Ceusters et al., 2005). In order to better define an ontology and avoid the concept misunderstanding, an ontology can be defined by several experts in the same or different domains (Acierno et al., 2017).

In many types of language structures for ontology, Ontology Web Language (OWL) is a language used for representing complex and rich knowledge of things, groups and relationships, etc. OWL is a language based on arithmetic logic that the model of knowledge built on OWL can be applied by the computer directly. Also, the model can be published to semantic web for browsing and further studying by other experts (W3C, 2012). The high interoperability is one of advantages of ontology (Pattuelli, 2011).

This research aims to clarify the component classes of historic buildings, the relationship between the classes and the properties of classes through the ontology model. After determining the necessary historical information, this model can be promoted to be used as a standard specification for Chinese historic buildings, thanks to its interoperability and computer readable semantic. Users can find out the hierarchy of components of historic building, the relationship between components and the historic information within the components.

\footnotetext{
${ }^{1}$ Autodesk Revit, https://www.autodesk.eu/products/revit-family/overview
} 


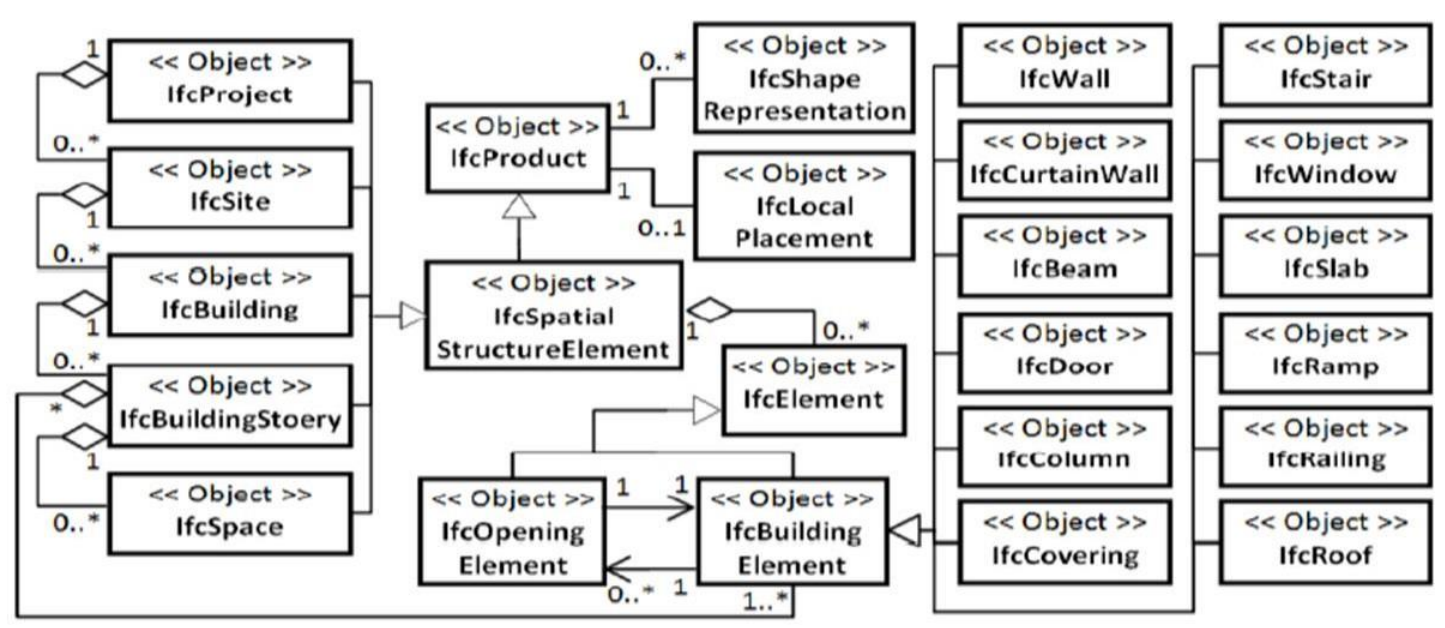

Figure 1. IFC building model (ISO 16739, 2011)

\subsection{Summary of literatures}

Nowadays it is common to apply digital archiving technology to the conservation of built heritage building. There are many researches and cases of using the Lidar or photogrammetry technology to build 3D models of heritage buildings, but HBIM is still a new domain in Taiwan. Therefore, some international researches were referenced for further studying. Murphy et al. (2009) suggested HBIM to be a new system for modelling the built heritage building. This research focuses on the modelling process of buildings such as the acquisition of point clouds, meshing and integrating additional properties. A parametric database based on historical data including crafts and decoration material was mentioned in the future work pf research which demonstrate the importance of the attribute of building components.

Brumana et al. (2013) modelled a built heritage building using terrestrial Lidar and photogrammetry. They considered the historical information of building, albeit only the $3 \mathrm{D}$ geometric model. For example, a wall which has the combination of different periods is separated into different models during the modelling. Every model has its own geometry attributes and historical information (the time of creation). The historical information concept is considered within HBIM in this research.

Dore \& Murphy (2012) extended HBIM to GIS by importing the model of built heritage building to CityGML. This made the management process no longer limited to the building itself but also the geometric information on its surroundings. The aim of this research is to combine the advantages of CAD parametric object and 3D GIS for assisting the analysis and conservation of built heritage building. The result shows that CityGML was not able to record the historical and geometry information of building in detail. It is mentioned that the next step is to build a database for storing, managing and browsing the attributes.

Fregonese et al. (2015) separated the model of built heritage building and the attributes of building. The attributes of building components were stored in a database for further edition and application. The software BIM3DSG was used to browse the 3D model and import the data from database. The attributes mentioned in this article are not historical information, only the geometric attributes of building components.

In conclusion, there are many related researches about HBIM which has mentioned the importance of historical information and how to make it coexist with 3D geometric model. But although many mentioned it in their future work or a small paragraph in the article, no detailed or complete method to deal this issue has been studied. It shows the value of developing a schema for conserving the historical and geometry information and presenting these data. Especially the lack of the literature in Taiwan shows that HBIM concept is not yet universal.

\section{METHODOLOGY}

\subsection{The data structure of building components}

It is important to investigate the building component and cultural information within built heritage building to apply HBIM to assist its conservation. For example, the building component and the information of component of a building in the Minnan style needed to be defined in detail before it become a component in software. The study case of this research is a Minnan style temple called Huangxi academy in Taichung, Taiwan. A traditional Minnan style building can be roughly separated into structural and non-structural components. The structural component is for carrying the weight of the roof or the building itself. From top to bottom, there are roof, wood structure, wall and foundation. The wood structure is the most complex structural component overall; it can be separated into rafter, beam, dougong, pillar and foundation of pillar. This research investigated the Huangxi academy by the report of the repair work done by Zeng et al. (2006). Beyond the building component, cultural information requirement was investigated through literature review of international heritage conservation charters and relational law in Taiwan. To summarise, this research defined a data structure for building components of a Minnan style building.

\subsection{Class-diagram and ontology of building component}

Class-diagram and ontology were established to represent and clarify the data structure of building component. The Unified Modeling Language (UML) was used in this research to establish the diagram. Due to the requirement of definition and 
classification method of a Minnan style building, the books Taiwan historic building graphic guide, Minnan traditional building and Taiwan traditional building: chapter of format and practice were also referenced. The structure of classdiagram was based on the design concept of Tsai (2009), Alhir (2003) and the IFC building model (ISO 16739, 2011).

The software used for establishing the ontology was Protégé 5.2.0 (Musen, 2015). It is a software for editing and acquiring knowledge which was developed by the Stanford University. Protégé has many plug-in functions and is one of the most widely used software for ontology editing. The language of ontology used was OWL as mentioned in section 2.3. The design concepts of both class-diagram and ontology were based on the data structure of the Minnan style building as seen in section 3.1. Individual building components were built in the ontology for demonstrating the design concept.

\subsection{Database of historical information}

The target of the database is the stakeholder who is in-charge of built heritage building, like artisans or conservation experts. For this purpose, the currently database in Taiwan does not satisfy the aspects of functions and information necessary. Taiwan has many historical information databases, for example the Huangxi academy's information can be found in the National Cultural Heritage Database Management System, the Cultural Heritage Department of Taichung City. However, the information within these databases are limited to some general description of the academy. There is no database recording a built heritage building in the scale of building component in Taiwan currently. Although more and more researches applied BIM to the conservation and management of built heritage building (Logothetis et al., 2015), the control and adaptability of BIM software is still less compared to the database approach in the aspect of data processing.

The convention of The United Nations Educational, Scientific and Cultural Organization (UNESCO) in 1972 has mentioned: monuments are architectural works and painting, elements or structures of an archaeological nature, inscriptions and combinations of features, which are of outstanding universal value from the point of view of history, art or science. The Cultural Heritage Preservation Act (2016) in Taiwan also stipulated: monuments are the buildings or affiliated facilities that were built to fulfil the needs of living and has value in history, culture and art. For a built heritage building, the year of creation is already an important historical information for it, since it presents the culture in that period from the paintings, decorations, building component, material and structure (Andaroodi and Kitamoto, 2010). In general, the historical information is a very important part of conservation.

Database is a collection of related data and data is a collection of facts and figures that can be processed to produce information. Most of the time data represents recordable facts. Data aids in producing information, which is based on facts. (Tutorials Point, 2015). Database types include single table database and relational database. The single table database will cost more memory space and has redundancy while dealing with complex and big data. This research chose relational database for storing the historical information. The software used was MySQL Workbench ver.6.3.6 and the data were from the reports of the Huangxi academy. The Huangxi academy has seen three major renovations, which were in 1986, 1999 and 2006 respectively. The structure of the database was based on the class-diagram.

\subsection{D model and integrated platform}

If the attribute of building component could not be arranged in its 3D model, the principal purpose of HBIM is lost. The 3D model not only has 3D surface but also geometry rules such as places, volume, material or the information in different construction period (Oreni et al., 2014). Current BIM software has the functions to build a model in the software environment. But the resulting model is worse than surveying technology when it comes to accuracy. If the model is not complex, the difference is small. However, most of the Minnan style buildings are full of decorations and sculptures. How to make the choice depends on the case encountered. The aim of this research is to establish a schema for HBIM for built heritage building in Taiwan. The feasibility of the schema is the main point of the research, not the accuracy of model. Therefore, the method of building 3D model of building component in this research is to build it directly in the software environment. The Autodesk Revit 2016 was chosen to create the 3D model. The 2D CAD drawings of Huangxi academy provided by the Center for Cultural Sites Rehabilitation and Development were the bases of 3D model. An example of a building component in 2D can be seen in Figure 2.

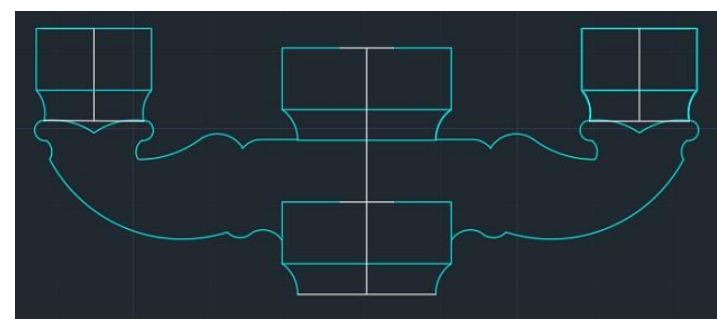

Figure 2. CAD drawing of a building component (Center for Cultural Sites Rehabilitation and Development, 2016)

After all parts of information are gathered, it is important to combine the information from different sources. The purpose of the integration platform was to combine the historical information and 3D model of building component so that the data can be browsed, shown and manipulated on the same platform. There are some functions for the platform according to the requirement of the stakeholder related to the heritage building management:

1. The platform can connect to the MySQL database and the $3 \mathrm{D}$ model.

2. Users can browse the tables in the database and the model in the BIM software.

3. The result of both information can be shown on the platform simultaneously.

4. The result of the browsing can be exported for further applications.

Autodesk Dynamo Studio ver. 1.2.1.3083 and its library Slingshot Package ver. 2014.11.30 were used for designing the platform. Dynamo Studio is a stand-alone programming environment that enables designers to create a visual logic to explore parametric conceptual designs and automate tasks 
(Autodesk Dynamo², 2017). Dynamo uses the concept of Node instead of programming directly which makes it easier to use and lots of libraries can be downloaded for different purposes.

\section{RESULTS AND DISSCUSIONS}

\subsection{The data structure of building components}

The references for data structure included Taiwanese Cultural heritage preservation act (2014), The Athens Charter (1931), The Venice Charter (1945), Nara Document on Authenticity (1994) and Operational guidelines for the implementation of the world heritage convention (2016). The resulting structure can be seen in Figure 3. The Timeline part means that every event of the component has to be recorded and presented in the format of timeline. In case of a mix of the events from different periods and therefore loses of the authenticity of historical information, a timeline is needed. The basic and cultural parts were the attributes of building components. Basic attributes were the geometry or physical information of the building component, which can be studied from the entity. The cultural information was the information from the literature or reports of the built heritage building, including the crafts of constructing the component, the tools used by carpenters, the carpenters who participated in the works, the user and usage of this building component and so on. For different styles of built heritage building, the cultural information can be redefined for better feasibility. The extend value part was the further study of the building component after the experts studied the relational information of the components. This data structure will be the basic of the class-diagram and ontology in the next section.

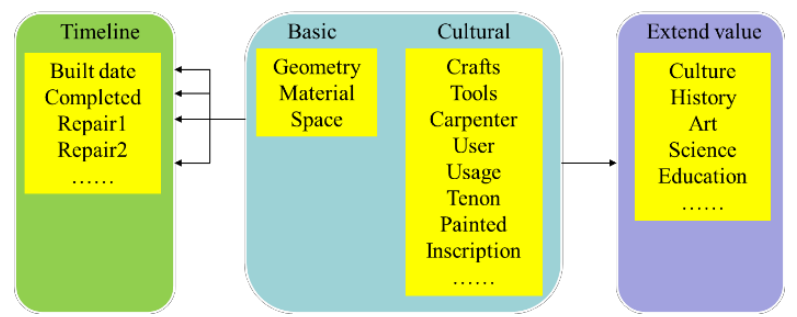

Figure 3. Data structure of building component

\subsection{The class-diagram of built heritage building}

The classes of the diagram was based on the data structure of the component and the target was Huangxi academy. There were several classes in the diagram:

1. Building: this class represents the whole building, including the basic information of the building. The information of a built heritage building is very rich, in this research only the most basic information is listed. For example, there were the name, address, management unit and relational person. Three classes composed this building class: component, surface treatment and structure class.

2 Structure: according to Li (2003), a Chinese built heritage building has different types of structures like roof, wood structure, wall and so on. The attributes for these structures were name, description, construction date, pattern etc. The construction date included every construction of this component in different periods and this attribute was related to a "Time" class, other classes that have the "construction date" attribute were like this. The pattern attribute was for the building's style. In Chinese built heritage building, even if the buildings are same style, there might be small different between different artisans, crafts.

The difference of structures is the value of attributes, so in this diagram the "structure code" class was built for distinguishing different structures. The structure code class was related to structure class by "type" attribute. In this case, redundancy classes would not be built for different types of structures.

The component classes were under the structure class. Different structures have different components. In this research, the dougong component of wood structure was chosen to demonstrate the component classes.

Figure 4 shows the organization of building, structure and code class.

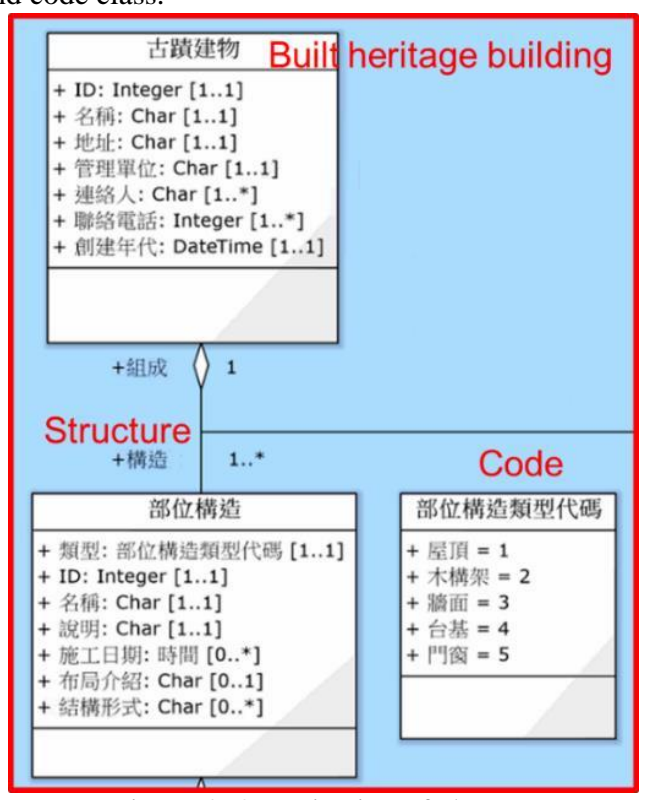

Figure 4. Organization of classes.

3. Dougong: this class represents the component that made the wood structure. The building component was the basic component in this research, in order to document the information in the scale of building component, these classes carried more attribute than others. The common attributes in building component class were style, name, ID, material, construction date, painting, artisan, tool, repair, description, craft and so on. Many common attributes have a related class, such as the material attribute related to material class for unified management.

Other than these classes, there were other classes for many different information types. There were 14 basic classes for a built heritage building and other class changes due to different buildings.

\footnotetext{
${ }^{2}$ Autodesk Dynamo, https://www.autodesk.com/products/dynamo-studio/overview
} 


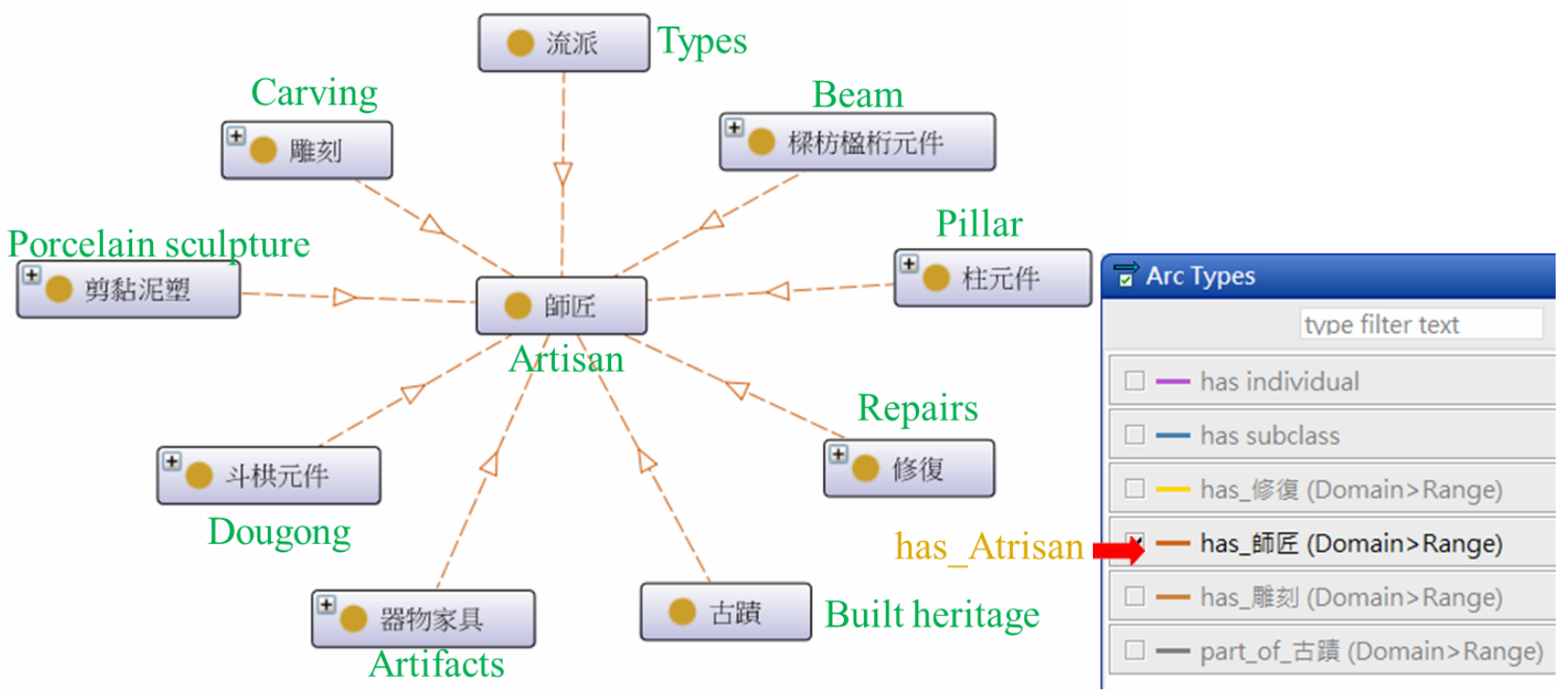

Figure 5. Object property of "has_artisan".

\subsection{The ontology of built heritage building}

The ontology was established on the OWL. The attributes of classes in OWL were the same as class-diagram. Data properties in OWL represent attributes and object properties represent the relationship between different classes. The object property "has_artisan" can be seen in Figure 5 for presenting the result of object properties. In the Figure 5, several classes have an object property value of "has_artisan" and they were related to the "artisan" class according to this property. This was set up in the domain of object property. In this way, every class can relate to other classes to fulfil the requirement of ontology.

In the Protégé environment, the OWL model can be uploaded to the semantic web for further applications. There are many ways to upload the ontology. The way of using GitHub and Ontoology was used in this research (Garijo et al., 2017). In this method, users upload their ontology files to GitHub and then fork the repositories to Ontoology. The Ontoology website will then upload the ontology file to the server of W3C in GitHub: W3id.org. In addition, the Ontoology produces many useful documents based on the ontology file such as evaluation of vocabularies and human readable ontology documents. These functions are very useful for further application and promote the interoperability of information. The URL of the ontology in this research is https://w3id.org/def/HXA. The URL will direct users to a human readable document of ontology if the URL is searched on the website or it will direct users to download the ontology if the URL is searched on the Protégé. To make sure that an ontology does not convey the wrong information, an ontology must be defined by many experts. In this research, the practical part of the ontology was for demonstrating the concepts of defining and publishing an ontology. Further studies and applications to Chinese built heritage building can refer to this schema.

\subsection{The database of built heritage building}

The result of database is shown in Enhanced EntityRelationship (EER) Diagram for better understanding of the tables and relationships within the database. EER diagram was produced after the database established, not like EntityRelation diagram which in general is used to show the blueprint before establishing a database. One of the functions called "reverse engineer" in the software MySQL Workbench 6.3 produced the EER diagram. Due to the complexity and big number of tables, a part of EER diagram was captured for demonstrating the organization of whole diagram.

Figure 6 shows the organization of the table for crafts information. The Brickwork and Carving were the sub-classes of crafts. The attribute "CraftsID" was foreign key for subclasses. The number of attributes was small since many attributes can be found in other tables and CraftsID relates these tables to Crafts class. In this way, although the artisans who used this craft cannot be seen in the Crafts table, it can be found after the browsing in database. This can fully use the advantage of relational database.

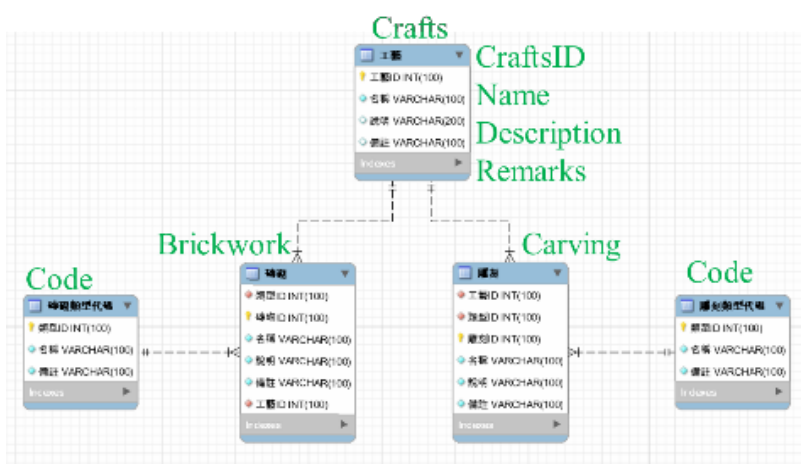

Figure 6. The Crafts part in the database.

The data in this database was from the renovation report of the Huangxi academy in 1986 and 2006. The data in the paper report was imported into the database according to its table. Some basic searching was done for evaluating the database. 
For example, if the user wants to find information about a component, the attributes of component will be shown.

\subsection{D model and integrated platform}

The resulting3D model of the building component was based on the 2D CAD drawing of Huangxi academy. Autodesk Revit 2016 was used to build the BIM model of the said building component. The result can be seen in Figures $7 \&$ 8. Figure 7 shows a 3D model of a special wood component of the building. After drawing the 3D surface, the attributes needed to be set up. The basic measurement attributes can be set up based on the data from the 2D CAD drawing. The material also needed to be added if the database in Revit does not match. After drawing all the components in the academy, the building can be built by using the components. Figure 8 shows the front gate of Huangxi academy.

According to the requirement of this research, the model from surveying technology was not used. Although the accuracy would be higher, the method to import surveying models into Revit is still needed to be further studied.

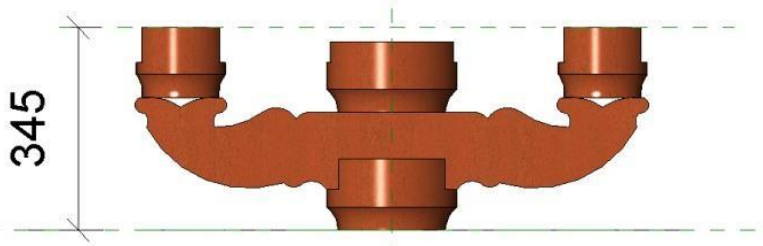

Figure 7. Example of a building component of the Huangxi academy.

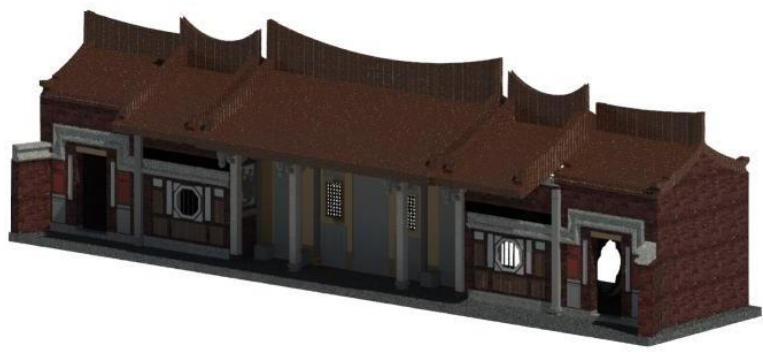

Figure 8. The front gate of Huangxi academy.

The integration platform was built in the Dynamo environment. The browsing result is shown in Figure 9. Users can browse the data in historical information database and the 3D model in Revit in the same time. The browsing result can be exported as a text file for further application.

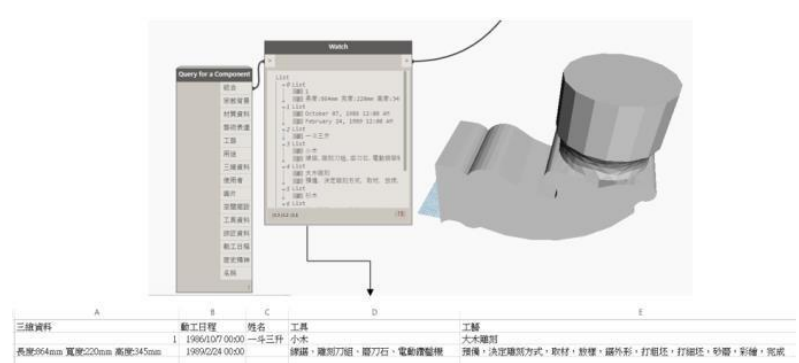

Figure 9. The browsing result of a building component in Dynamo.

\subsection{Discussion}

The class-diagram and ontology model of built heritage building can be further studied to become a prototype of standard for Chinese built heritage. It is nearly impossible to define a frame that is suitable for every Chinese building. However, the concept of this research can be applied to other relational studies. Even in the building style is different; the users only need to make some adjustment in order to use it.

Browsing results of the database were based on the possible requirements of the stakeholders and the interview of the person who frequently contacts the artisans of the built heritage building. The prove of applicability will be stronger if the opinions of artisans and experts of conservation can be included. The current result shows that the database can improve the speed and accuracy of browsing.

The integrated platform can provide the 3D model and historical information of the building component. Although the characteristic is different, the platform improved the functionality of the database and model management. The limit of this platform is that if the model was not produced within the Autodesk environment, there is a possibility that the platform cannot show the model.

\section{CONCLUSIONS \& SUGGESTIONS}

The concept of HBIM for Chinese built heritage building in Taiwan is still a fairly new concept. This research was a prototype of the HBIM schema for testing its feasibility for assisting the conservation of built heritage building. The result of practical works show that establishing a class-diagram or ontology can help to clarify the organization concept of Chinese built heritage building. In addition, the ontology of buildings can increase the interoperability of information, since every computer can understand the structure of the ontology model. This advantage makes it easier to apply the schema for different buildings. The integration platform has been proven to be better than the traditional recording method. If the conservation process can be recorded in detail into the database, the authenticity of the building could be conserved completely. However, the target user of this integration platform is the stakeholders of built heritage building.

Huangxi academy is the only case study of this research, as mentioned in the discussion; the feasibility of this schema to be implemented for other styles of Chinese built heritage building needs more case studies in order to support it.

The 3D models of the building components of Huangxi academy was built within the Revit environment. If the model from surveying technologies can be applied to BIM environment, the accuracy of the model will be increased while keeping the faithfulness of the object geometry. If the model from photogrammetry or laser scanning can be imported to BIM environment perfectly, the schema can be used to faithfully conserve the object and will be useful for the stakeholders in built heritage building. 


\section{REFERENCES}

Acierno, M., Cursi, S., Simeone, D., Fiorani, D., 2017. Architectural heritage knowledge modelling: An ontologybased framework for conservation process. In Journal of Cultural Heritage, 24, pp. 124-133.

Alhir, S., 2003. Learning Uml. " O'Reilly Media, Inc." p 256.

Andaroodi, E., Kitamoto, A., 2010. Architectural heritage online: Ontology-driven website generation for world heritage sites in danger. In Lecture Notes in Computer Science, 6436, pp. 277-290.

Brumana, R., Oreni, D., Raimondi, A., Georgopoulos, A., Bregianni, A., 2013. October. From survey to HBIM for documentation, dissemination and management of built heritage: The case study of St. Maria in Scaria d'Intelvi. In Digital Heritage International Congress (DigitalHeritage), 1 , pp. 497-504.

Ceusters, W., Smith B., Goldberg L., 2005. A terminological and ontological analysis of the NCI Thesaurus, In Methods of Information in Medicine, 44(4), pp. 498-507.

Dore, C., Murphy, M., 2012, September. Integration of Historic Building Information Modeling (HBIM) and 3D GIS for recording and managing cultural heritage sites. In Virtual Systems and Multimedia (VSMM), pp. 369-376.

El-Mekawy, M., Ö stman, A., Hijazi, I., 2012. A unified building model for 3D urban GIS. In ISPRS International Journal of Geo-Information, 1, pp. 120-145

Fregonese, L., Achille, C., Adami, A., Fassi, F., Spezzoni, A., Taffurelli, L., 2015. BIM: An integrated model for planned and preventive maintenance of architectural heritage. In Digital Heritage, 2, pp. 77-80

Fu, C. C., 2005. The authenticity and maintain of conservation for world cultural heritage. National Cheng Kung University.

Garijo, D., Ruiz-Iniesta, A., Poveda, M., Corcho, O., Garcia, M. A., 2017. WIDOCO 1.4.1. Zenodo. Retrieved October 6 from http://doi.org/10.5281/zenodo.583745.

Hsieh, S. H., Kuo, J. C., Chen, H. T. and Tsai, Y. H., 2014. Learning BIM through Project Exercise: Basics (Revised Edition). National Taiwan University Press. p 185.

ICOMOS, 1931. The Athens Charter for the restoration of historic monuments Carta del Restauro 2 \& general principles. In 1st International Congress of Architects and Technicians of Historic Monuments, Athens.

ICOMOS, 1964. International charter for the conservation and restoration of monuments and sites Article 2, In Second international congress of architects and technicians of historic monuments, Venice.

ICOMOS, 1994. Nara Document on Authenticity Article 11. Nara, Japan.

Li, C. L., 2003. Taiwan historic building graphic guide. YuanLiou Publishing Co. Ltd. p 359.
Lin, H. C., 1987. Taiwan traditional building: chapter of format and practice. Artistpublishing. p 224.

Logothetis, S., Delinasiou, A., Stylianidis, E., 2015. Building Information Modelling for Cultural Heritage: A review. In ISPRS Annals of Photogrammetry, Remote Sensing and Spatial Information Sciences, II-5/W3, pp. 177-183.

Murphy M., McGovern E., Pavia S., 2007. Parametric Vector Modelling of Laser and Image Surveys of 17th Century Classical Architecture in Dublin, In The 8thInternational Symposium on Virtual Reality, Archaeology and Cultural Heritage VAST, pp. 27-29.

Murphy, M., McGovern, E. Pavia, S., 2009. Historic building information modelling (HBIM), In Structural Survey, 27(4), pp. $311-327$.

Musen, M. A., and the Protégé Team., 2015. The Protégé Project: A Look Back and a Look Forward. AI Matters, pp. 4 12

Oreni, D., Brumana, R., Georgopoulos, A., Cuca, B., 2013. HBIM for Conservation and Management of Built Heritage: Towards a Library of Vaults and Wooden Bean Floors. In ISPRS Annals of Photogrammetry, Remote Sensing and Spatial Information Sciences. II-5/W1, pp. 215-221.

Oreni, D., Brumana, R., Georgopoulos, A., Cuca, B., 2014. HBIM Library Objects for Conservation and Management of Built Heritage. In International Journal of Heritage in the Digital Era, 3(2), pp. 321-334.

OWL Working Group, 2012. "Web Ontology Language (OWL)," W3C Semantic Web, [Online]. Retrieved October 6, 2017, from https://www.w3.org/OWL/.

Pattuelli, M. C., 2011. Modeling a domain ontology for cultural heritage resources: A user-centered approach. In Journal of the American Society for Information Science and Technology, 62(2), pp. 314-342.

Tsai, H. L., 2009. Object-Oriented Analysis and Design with Applications (Third Edition). GOTOP INFORMATION INC. p 692.

Tsao, C. P., 2006. Minnan traditional building. Xiamen University. p 316.

Tutorials Point. (n. d.) DBMS database management system Retrieved October 16, 2017, from http://www.kciti.edu/wpcontent/uploads/2017/07/dbms_tutori al.pdf .

UNESCO, 2008. Operational Guidelines for the Implementation of the World Heritage Convention Article 82. Paris.

Yen, Y. N., 2014. Introduction to Digital Cultural Heritage Resources in Taiwan. Bureau of Cultural Heritage Ministry of Culture. p 40.

Zeng, W. C., 2006. Report of repairing project of Huangxi academy in roof. Taichung City Government. 